\title{
Neurofeedback Intervention for Emotional Behavior Regulation in Schizophrenia: New Experimental Evidences from Optical Imaging
}

\author{
Michela Balconi ${ }^{1,2^{*}}$ and Maria Elide Vanutelli2,3 \\ ${ }^{1}$ Department of Psychology, Catholic University of Milan, Milan, Italy \\ ${ }^{2}$ Research Unit in Affective and Social Neuroscience, Catholic University of Milan, Milan, Italy \\ ${ }^{3}$ Department of Philosophy, Università degli Studi di Milano, Milan, Italy
}



\section{Introduction}

Schizophrenia (SZ) is a chronic and severe neuropsychiatric syndrome that affects reasoning, feelings, and behaviors, with a substantial social and relational dysfunction. In recent years, researchers from the neuroscientific field tried to identify some biological markers and neural correlates which could represent the symptoms underlying SZ deficits and support the diagnosis (Linden \& Fallgatter, 2009) that is usually made by observable symptoms. Such research engaged patients in different experimental protocols and recorded their neural activity by using different neuroimaging techniques like functional magnetic resonance imaging (fMRI), positron emission tomography (PET), and, more recently, functional near-infrared spectroscopy (fNIRS). A relatively new technique, fNIRS allows a photometrical measurement of changes in the concentration of oxy $(\mathrm{O} 2 \mathrm{Hb})$ and deoxyhemoglobin $(\mathrm{HHb})$ in brain tissue (Jobsis, 1977). Compared to other functional neuroimaging techniques, fNIRS has some disadvantages, such as a lower spatial resolution and the inability to reach deep brain regions. However, it was successfully applied in studies involving neuropsychiatric patients, since it is portable and can be easily and noninvasively positioned in naturalistic environments (even at the 
bedside). More importantly, it doesn't impose movement limitation and it is completely silent. That's why it became especially popular in research with patients suffering from SZ spectrum disorders.

Previous fNIRS research with SZ patients mainly focused on cognitive impairment, by assessing verbal fluency (Ehlis, Herrmann, Plichta, \& Fallgatter, 2007; Kubota et al., 2005; Takizawa et al., 2008), divergent thinking (Takeshi, Nemoto, Fumoto, Arita, \& Mizuno, 2010) and insight (Pu et al., 2013), as well as attentive (Shoji et al., 2013) and executive functions (Zhu et al., 2010). The results of these studies consistently showed reduced prefrontal activation during the cognitive tasks in SZ patients (functional hypofrontality) compared to healthy controls (see also Shinba et al., 2004; Watanabe \& Kato, 1999). Moreover, instead of overall activation effects in SZ patients, Falgatter and Strik (2000) found a specific hemispheric effect with a lack of lateralized activity, interpreted as a sign of reduced specific lateralized frontal reactivity.

However, prefrontal and frontal regions are also core regions during emotional/social regulation. As pointed out by Doi and colleagues (Doi, Nishitani, \& Shinohara, 2013), fNIRS is really suitable at quantifying emotional functioning in the prefrontal cortex, which is also significantly impaired in SZ patients. In fact, although positive symptoms usually tend to respond to pharmacological treatment, negative symptoms tend to persist in the form of affective flattening, alogia, anhedonia, asociality and emotion dysregulation. As pointed out by Balconi and colleagues (Balconi, Tirelli, \& Frezza, 2015), emotional deficits in $\mathrm{SZ}$ affect different processes, such as emotional experience (Taylor et al., 2012), expression (Blanchard \& Cohen, 2006), and recognition (Horan et al., 2012). Of course, this is particularly important, since these symptoms affect patients' personal and interpersonal dimensions, as well as their social functioning. Despite the significant interest of this topic, few previous research applied neuroimaging techniques to explore emotional dysfunction in SZ patients (for a review see Balconi, Tirelli, et al., 2015). For example, Egashira and colleagues (Egashira et al., 2015) found that SZ patients showed less activation in the superior frontal, orbital frontal, and middle temporal regions during an emotional go/no-go task compared to healthy subjects. In addition, Pu and colleagues (Pu et al., 2016) explored the relation between frontal activity during a working memory task and social cognition in SZ patients. The authors found the presence of a significant relation between the activity within the lateral PFC and theory of mind scorings.
Interestingly, some other studies demonstrated how the brain activity is associated with SZ severity and with some symptoms that affect patients' everyday life. For example, Nishimura and colleagues (Nishimura et al., 2011) found that the abnormal activity over prefrontal and frontopolar regions during response inhibition is associated with excitement symptoms. Also, Watanabe and colleagues (Watanabe, Urakami, Hongo, \& Ohtsubo, 2015) found that $\mathrm{O} 2 \mathrm{Hb}$ values within the frontal areas reflect disease severity and the degree of social adjustment. In fact, patients with increased frontal activation were better socially adjusted than those with lower responses.

However, another important issue about SZ research that should be addressed by the neuroscientific approach is related to the assessment of treatment effects. In fact, although imaging techniques are contributing to the knowledge of the impaired frontal networks in SZ in both cognitive and emotional domains, the clinical applications in treatment monitoring are still inconsistent. Nonetheless, considering the resistance of emotional and social symptoms to the pharmacological treatment, there is the urgency to validate and develop new interventions based on objective data in support of more conventional and subjective outcome measured according to patients' reports. In recent years, neurofeedback (NF) was introduced as an innovative method to improve patients' awareness about some cognitive or emotional symptoms. In fact, as a behavioral approach by operant conditioning paradigm and shaping procedure (Sherlin et al., 2011), it can reinforce voluntary control on some forms of neural activity, such as the electrophysiological modulation, by eliciting desirable brain waves and inhibiting abnormal responses (Kouijzer, van Shie, de Moor, Gerrits, \& Buitelaar, 2010). The efficacy of NF on SZ has been demonstrated starting from the pioneering studies (Balconi, Frezza, \& Vanutelli, 2018; Schneider et al., 1992), in which attention breakdown of schizophrenic patients was improved by regulating the slow cortical potentials. Also, in an in-depth case study, Schummer (2008) uncovered favorable effects of NF in executive functioning. Similar results have been also found by Naimijoo and colleagues (Naimijoo, Rezaei, \& Feizzadeh, 2015) on a larger sample. Another examples of NF application to the cognitive domain in SZ is the work of Surmeli and colleagues (Surmeli, Ertem, Eralp, \& Kos, 2012), which showed increased attentive scorings after the NF intervention, as well as improved symptoms severity. In addition, a single-case intensive training conducted by Nan and colleagues (Nan et al., 2017) showed an 
improvement in short-term memory, mood, and speech after NF.

For what concerns the emotional domain, instead, Ruiz and colleagues (Ruiz et al., 2013) trained nine schizophrenic patients to up- and downregulate anterior insular cortex activity by fMRI neurofeedback to improve face emotion recognition. Moreover, by following a specific theoretical model on emotion processing lateralization, Gruzelier and colleagues (Gruzelier, Hardman, Wild, \& Zaman, 1999) trained patients to shift EEG negativity away from their functionally dominant hemisphere. Specifically, they induced a rightward direction in the Active syndrome with left-sided functional biases, and a leftward direction in the Withdrawn syndrome with right-sided functional biases.

However, the existing studies are only preliminary and generally not exhaustive in term of experimental paradigm (no imaging evidences after NF treatment; Naimijoo et al., 2015), sample size (only single or few cases; Schummer, 2008) or specific type of treatment. Thus, the aim of the present study was to evaluate the efficacy of a NF intervention to improve emotional regulation in a pilot sample of SZ patients. The procedure included an initial assessment during a passive emotional task (T0) to explore the brain mechanisms related to emotional processing before and after the training (pre/post) by means of fNIRS covering the frontopolar area. Together with stimuli presentation, the explicit subjective evaluation of emotional stimuli was assessed. Then, a NF intervention was planned and performed for the following 5 weeks (T1). It consisted in an emotional training by the modulation of delta-theta EEG range. Following the same idea of Gruzelier and colleagues (Gruzelier et al., 1999) about lateralization enhancement, we trained patients to intensify such range in the less responsive hemisphere, based on a previous assessment. It was proposed only to a group of patients assigned to the experimental (E) group, while the other half was assigned to the control (C) group.

Subsequently, the emotional assessment was repeated (T2) exactly as in T0. According to previous research (Kring \& Moran, 2008) we expected preserved capacity to discriminate stimulus valence yet increased in T2 for the E group. For what concerns the neural level, instead, we expected a significant effect of the training over prefrontal brain activity for the $E$ group in T2, which could also be more lateralized according to stimuli valence (Fallgatter \& Strik, 2000; Balconi, Grippa, \& Vanutelli, 2015a; Balconi, Vanutelli, \& Grippa, 2017).

\section{Materials and Methods}

\section{Participants}

The study recruited a pilot sample of 25 institutionalized patients, 12 females (Mage $=32.10$; $S D=4.76$; range $=28-40$ ). The sample is an extension of a preliminary data acquisition on 19 patients (see Balconi, Frezza, et al., 2018). Establishment of diagnoses was based on semistructured interviews which were conducted by an expert psychiatrist according to the criteria of the fourth edition of the Diagnostic and Statistical Manual of Mental Disorders (SCID-I: Structured Clinical Interview for DSM IV Axis I Disorders; American Psychiatric Association, 2000). Informed consent was obtained after full explanation of purpose and procedure of the study. Inclusion criteria were: (1) age between 18 and 45 years old; (2) fair psychopathological compensation; (3) stable pharmacological program followed for at least 4 weeks before the beginning of the study. Exclusion criteria were as follows: (1) visual or auditory impairment; (2) concurrent substance abuse (abstinence for at least 3 months); (3) Diagnosis of moderate or severe mental retardation (IQ < 55); (4) neurological damage; (5) anamnesis of brain injury.

Then, patients have been randomly assigned to either the $C$ group, composed of 11 patients, 6 females $\left(M_{\text {age }}=34.78 ; S D=5.04\right.$; range $\left.=27-40\right)$, or the neurofeedback (NF) group, composed of 14 patients, 6 females $($ Mage $=35.23 ; S D=4.09$; range $=28-38)$. The two groups were comparable in term of emotional intelligence (as assessed by the Mayer-SaloveyCaruso Emotional Intelligence Test, MSCEIT), cognitive competencies (as assessed by the Wechsler Adult Intelligence Scale Fourth Edition, WAIS-IV) and neuropsychological profile (as assessed by the Brief Assessment of Cognition in Schizophrenia, BACS). Patients in the $\mathrm{C}$ group followed the Treatment-As-Usual (TAU), while patients in the NF group followed both TAU and NF training.

The study included a first phase of assessment (T0), the NF intervention (T1), and a second assessment (T2) to evaluate the treatment efficacy. Procedures in T0 and T2 were identical to allow direct comparison of their measures (for the original protocol, see Balconi, Cotelli, et al., 2018). The assessment consisted of the recording of hemodynamic parameters by means of fNIRS during a passive emotional task, and of subjective ratings in terms of valence and arousal of stimuli, by means of the SelfAssessment Manikin (SAM; Russell, 2003). 
The study and its procedures followed the principles of the Declaration of Helsinki and were approved by the Ethics Committee of the Department of Psychology of the Catholic University of the Sacred Heart. Written informed consent for the enrollment of patients included in the experimental cohort was obtained from their legal representatives.

\section{Stimuli}

Patients were required to observe and then evaluate affective patterns at the end of the stimuli processing. One hundred pictures were taken from the International Affective Picture System (IAPS) (Bradley \& Lang, 2007), depicting 40 positive and 40 negative pictures (20 low and 20 high arousing, each), and 20 neutral stimuli, based on valence and arousal ratings obtained from a prior validation study (Balconi, Brambilla, \& Falbo, 2009). IAPS subjective ratings were obtained with the SAM scale, using an easier adapted 5-point version (Bradley \& Lang, 2007). For IAPS codes, see (Balconi, Grippa, \& Vanutelli, 2015b).

\section{Procedure}

Patients were seated in a dimly lit room, facing a computer monitor that was placed $70 \mathrm{~cm}$ from the subject. The stimuli were presented using STIM2 software (Compumedics Neuroscan, Charlotte, NC) running on a personal computer with a 15 -in. screen. Participants were required to observe each stimulus during fNIRS recording, and they should attend to the images the entire time of exposition. Pictures were presented in a random order in the center of a computer monitor for $6 \mathrm{~s}$, with an inter-stimulus interval of $12 \mathrm{~s}$. 120-s eyes-closed and 120-s eyesopen resting baselines were registered at the beginning of the experiment before the picture series. After the experimental phase, patients had time to rate their emotional experience on the SAM scale evaluating valence and arousal on a bipolar scale applied to each picture.

\section{fNIRS Recording and Analysis}

fNIRS measurements were conducted with a NIRScout system (NIRx Medical Technologies, LLC, Los Angeles, CA) using a 6-channel array of optodes (4 light sources/emitters and 4 detectors) covering the prefrontal area. Emitters were placed on positions AF3-AF4 and F5-F6, while detectors were placed on AFF1-AFF2 and F3-F4 (see Figure 1). Emitterdetector distance was $30 \mathrm{~mm}$ for contiguous optodes and a near-infrared light of two wavelengths (760 and $850 \mathrm{~nm}$ ) was used. With NIRStar (NIRScout acquisition software), changes in the concentration of oxygenated $(\mathrm{O} 2 \mathrm{Hb})$ and deoxygenated hemoglobin $(\mathrm{HHb})$ were recorded continuously throughout the paradigm. Signals obtained from the 6 NIRS channels were measured with a sampling rate of 6.25 $\mathrm{Hz}$, analyzed and transformed with nirsLAB software (v2014.05; NIRx Medical Technologies, LLC, Glen Head, NY), according to their wavelength and location, resulting in values for the changes in the concentration of $\mathrm{O} 2 \mathrm{Hb}$ and $\mathrm{HHb}$ for each channel. The raw data of $\mathrm{O} 2 \mathrm{Hb}$ and $\mathrm{HHb}$ from each channel were digitally band-pass filtered at $0.01-0.3 \mathrm{~Hz}$. Then, the mean concentration of individual channel was calculated by averaging data across trials from the trial onset for $6 \mathrm{~s}$. The mean concentration value of 6 $s$ immediately before each trial was used as eventrelated baseline. Based on the mean concentrations in the time series, we calculated the effect size in every condition for each channel within a subject, calculated as the difference of the means of the baseline ( $\mathrm{m} 1)$ and trial (m2) divided by the standard deviation (s) of the baseline: $d=(m 1-m 2) / s$. Then, the effect sizes obtained from the 6 channels were averaged in order to increase the signal-to-noise ratio. These normalized effect sizes could be averaged regardless of the unit (for this procedure, see Balconi, Grippa, et al., 2015a, 2015b; Balconi, Vanutelli, Bartolo, \& Cortesi, 2015). To interpret the eventrelated responses to stimuli with respect to each baseline, signs have been inverted. 


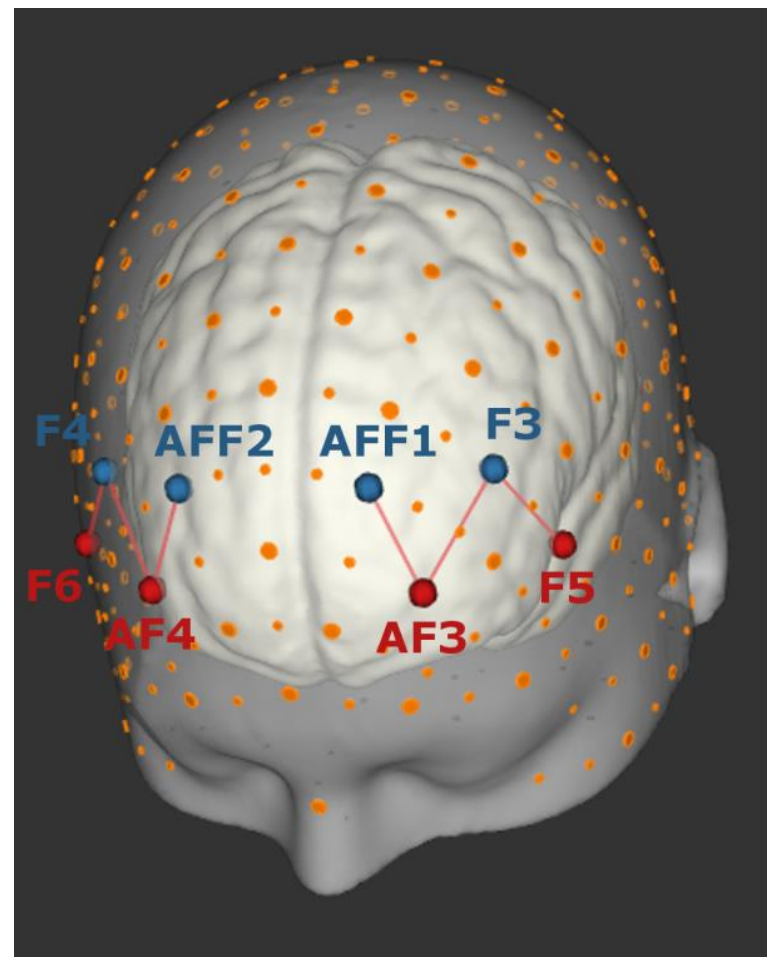

Figure 1. Location of NIRS sources (red) and detectors (blue) over the frontopolar areas.

\section{Neurofeedback Training}

The training consisted in a 5-week successive training period. The experimental group completed 10 sessions, each lasting approximately 25 minutes with 2 intervals of $2 \mathrm{~min}$ in between. The sessions were performed in the same room as the assessment phases. NF was administered using a ProComp2 device (Thought Technology Ltd, Montreal, Canada) and the task was created and presented by BioGraph Infiniti software package (Thought Technology Ltd, Montreal, Canada). Before each training session, a 2-min resting baseline was recorded with eyes open. Patients were instructed to reduce muscle activity and control eye blinks. EEG was recorded by placing an electrode in correspondence to the left (F3) or the right hemisphere (F4), according to each patient's electrophysiological pattern as assessed in a preliminary session. Thus, training was lateralized as a function of preliminary patient's baseline left or right higher activity. The reference was placed on the contralateral earlobe, and ground electrode on the ipsilateral earlobe. The main visual feedback and reward tool was a video made up of IAPS pictures different from those used during the initial assessment phase: when the EEG band of interest reached values over the established rewardthreshold, the video proceeded and showed different affective stimuli. Thus, patients had to learn to enhance the low-frequency range within the less active hemisphere. The reward threshold is automatically managed by the software such that a fixed $80 \%$ reward level is provided. The band values were as follows: reward $=0.5-5.5 \mathrm{~Hz}$; inhibit-low = $0.1-0.5 \mathrm{~Hz}$; inhibit-high $=50-64 \mathrm{~Hz}$.

\section{Results}

\section{SAM Ratings}

Arousal and valence subjective scorings (dependent measures) were analyzed with two separated three repeated factor ( 2 arousal; 3 valence; 2 time) and one between factor (2 group, C group vs. NF group) mixed-model ANOVAs. For all ANOVA tests, degrees of freedom were corrected by GreenhouseGeisser epsilon where appropriate. Moreover, due to multiple independent analyses and comparisons, we applied Bonferroni test for inequality. Contrast analyses (paired comparisons) were applied to significant main or interactions effects.

For valence ratings, Valence main effect was significant $(F(2,49)=8.13, p<.01)$. Indeed, negative valence stimuli received significantly $(p<.01)$ lower values $(M=1.86 ; S D=0.03)$ than positive stimuli $(M$ $=3.84 ; S D=0.41$ ), with intermediate level for neutral ones $(M=2.27 ; S D=0.34)$, which were significantly higher $(p<.01)$ than negative, and lower $(p<.01)$ than positive pictures. Also, Time main effect was significant $(F(1,24)=7.45, p<.01)$, with increased/decreased values based on valence for all conditions in T2 (for positive $M=3.77$; $S D=0.18$; for negative $M=2.40 ; S D=0.16$ ) than T0 (for positive $M$ = 3.02; $S D=0.09$; for negative $M=1.74 ; S D=0.10$ ). Finally, the Valence $\times$ Time $\times$ Group interaction effect was significant, with negative pictures evaluated as more positive $(p<.05)$ by NF group in T2 $(M=2.88$; $S D=0.13)$ than T0 $(M=1.65 ; S D=0.12)$ and positive pictures evaluated as more positive $(p<.01)$ by NF group in T2 $(M=3.80 ; S D=0.18)$ than T0 $(M=1.84$; $S D=0.10)$. In contrast, $C$ group did not show this significant effect. For arousal ratings, instead, no significant effect emerged (see Figure 2). 


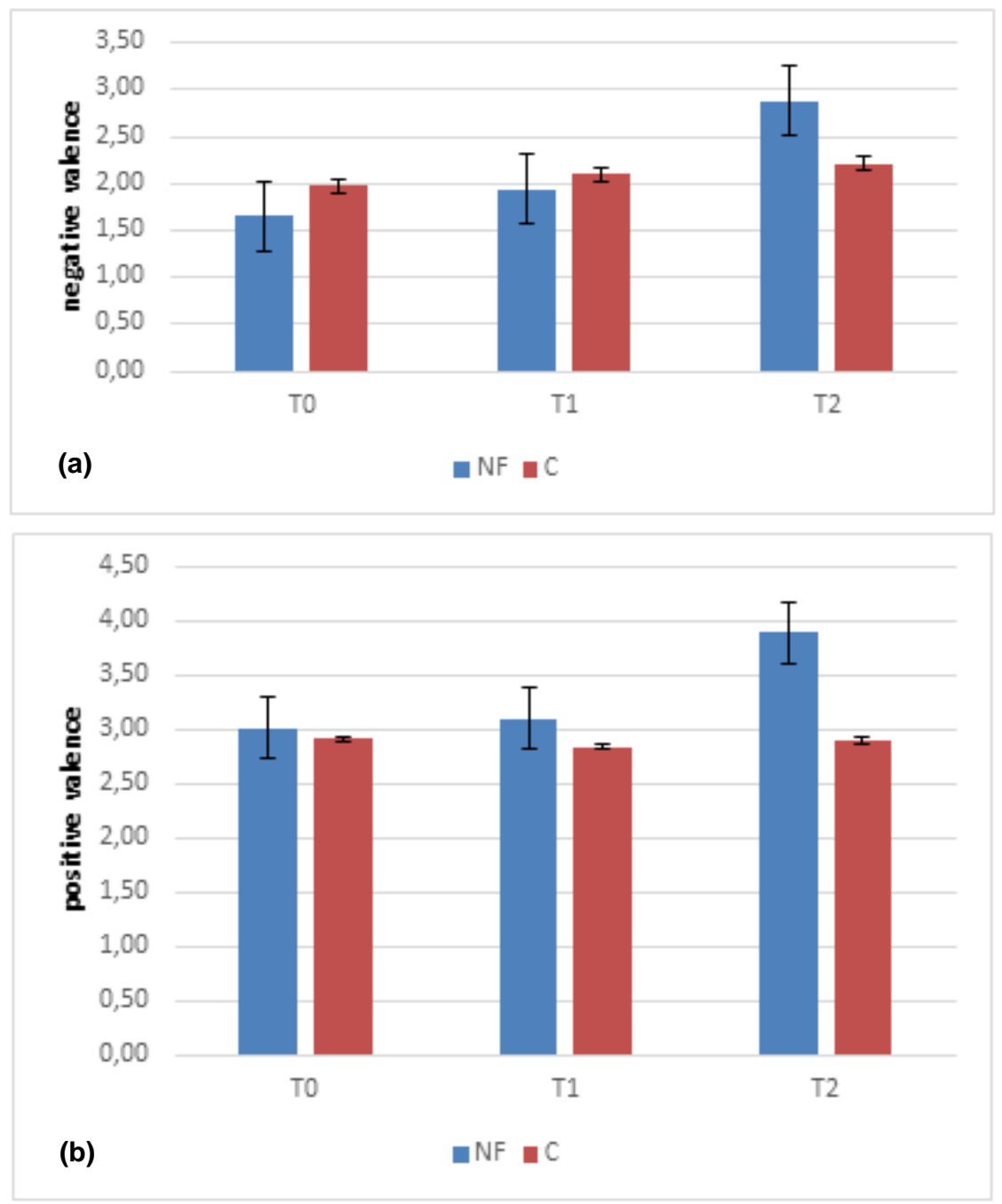

Figure 2. SAM ratings as a function of group, valence and time for (a) negative and (b) positive pictures.

\section{fNIRS Results}

About fNIRS recordings, repeated measure ANOVAs were applied with respect to $D$ dependent measure of $\mathrm{O} 2 \mathrm{Hb}$ concentration in all the channels (Ch1-Ch6). Successively, for a second set of analysis data were averaged considering left (Ch1: AF3-F3; Ch2: AF3AFF1; Ch3: F5-F3) and right (Ch4: AF4-F4; Ch5: AF4-AFF2; Ch6: F6-F4) regions to obtain an inclusive index based on the specific lateralized NF application.

The first set of analysis was aimed at assessing the effect of figure valence on brain activation before and after NF (Group $\times$ Valence $\times$ Channel $\times$ Time) and showed a significant effect for figure valence $(F(2,48)$ $=8.78, p<.01$ ), with increased activity for positive and negative that neutral stimuli (Figure 3 ). In addition, Group $\times$ Valence $\times$ Time $\times$ was significant $(F(2,48)=8.13, p<.01)$, with increased activity for

NF compared to $C$ for positive $(F(1,28)=6.90, p$ $<.01)$ and negative $(F(1,48)=7.34, p<.01)$ stimuli in T2. No significant effect was found for arousal.

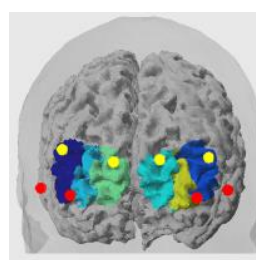

NEUTRAL

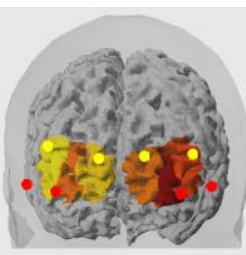

POSITIVE

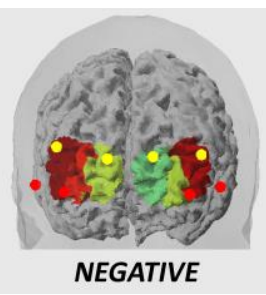

Figure 3. Projection of raw $\mathrm{O} 2 \mathrm{Hb}$ activation in response to neutral (left), positive (middle), and negative (right) pictures from one exemplificative subject. 
The second set of analysis was aimed at assessing the lateralized hemodynamic effect (Group $\times$ Valence $\times$ Lateralization $\times$ Time) before and after NF. Results showed a significant Group $x$ Valence $\times$ Lateralization effect $(F(2,48)=8.55, p<.01)$. Indeed, the NF group presented increased $\mathrm{O} 2 \mathrm{Hb}$ levels in $\mathrm{T} 2$ within the right side in response to negative stimuli
$(F(1,48)=7.13, p<.01)$ compared to the $C$ group. In addition, $\mathrm{NF}$ group showed increased $\mathrm{O} 2 \mathrm{Hb}$ levels in T2 within the left side in response to positive stimuli $(F(1,48)=7.98, p<.01)$ compared to the $C$ group (see Figure 4). No significant effect was found for arousal.



Figure 4. $\mathrm{O} 2 \mathrm{Hb}$ activation as a function of group, valence and time for positive (a) and negative (b) pictures.

\section{Discussion}

The present research aimed at investigating the effects related to a NF training over the prefrontal neural activity in a small sample of SZ patients. The experiment was subdivided into three different sections, with the neurophysiological assessment being proposed at $\mathrm{T} 0$ and $\mathrm{T} 2$, and the NF training administered in between (T1) and only to the experimental $(E)$ group. The training was meant to provide the patients the capacity to self-regulate their own cortical activity while processing different affective pictures. The study highlighted different significant results both at a behavioral and a neurophysiological level.

For what concerns valence and arousal ratings, the analysis showed that patients of both groups were 
able to correctly identify the hedonic valence of IAPS stimuli, both in T0 and T2. In fact, they identified negative stimuli as more negative than positive ones, and vice versa. This result is in line with previous research (Balconi, Frezza, et al., 2018, Kring \& Moran, 2008) that identified preserved attributional processes in SZ patients. Indeed, it is possible to argue that this capacity does not depend on the NF training. However, a significant interaction effect showed that both negative and positive stimuli received more positive values in T2 compared to T0 only in the E group. This specific enhancement effect towards positive feelings could, instead, be attributed to the NF training as a consequence of an alleged more functional management of negative effects (Balconi, Tirelli, et al., 2015). On the other hand, patients faced more difficulties in discriminating the arousing power of pictures, which could derive from an impairment in detecting the motivational significance of external stimuli (Williams et al., 2004). At this regard, future research could better investigate this issue by using a combined bio + neurofeedback to provide a clearer signal to be modulated by the arousal dimension.

For what concerns the neural level, instead, a first result revealed that, irrespective of group and time, the $\mathrm{O} 2 \mathrm{Hb}$ levels increased over the frontopolar regions for positive and negative stimuli if compared to neutral ones. This result is in line with previous work (e.g., see Balconi \& Vanutelli, 2016, 2017; Herrmann et al., 2008) assessing emotional pictures as being more activating than neutral ones. Such a mechanism could be interpreted as finalized to alert the emotional behavior in response to the highly significant emotional stimuli patients are interacting with (Balconi \& Vanutelli, 2016). Moreover, this is also in line with the behavioral result confirming patients' preserved ability to detect hedonic valence.

In addition, a significant interaction revealed a higher activity for $\mathrm{E}$ compared to $\mathrm{C}$ group for positive and negative stimuli in T2. Fallgatter and colleagues (Fallgatter \& Strik, 2000) found that, when performing a cognitive task, SZ patients did not display the significant increase in hemispheric asymmetry that was instead evident in controls. Since patients' and controls' behavioral performance did not differ, the author concluded that the absence of this lateralized activation couldn't be attributed to a dysfunctional strategy and interpreted such effect as a different functional response from patients who lacked lateralized activation. Similarly, in our case, although patients showed a congruent hemodynamic response to emotional stimulation (both positive and negative valenced stimuli), the effect of the NF training could have been in the direction of an improved awareness during emotion processing, which resulted in a more positive emotional attribution, as also revealed by SAM data. However, future studies could better explore this issue by administering further questionnaires to assess patients' emotional experience, to be acquired also at long-term distances.

To conclude, as pointed out by the present results, the use of imaging methods such as fNIRS could provide significant evidence about the efficacy of an innovative and noninvasive training such as NF, which could anyhow provide patients with the capacity to self-regulate their own cortical activity during emotion processing. At present, however, since we did specifically acquire data about the microvolt levels for the frequency over time, we did not consider the learning trend during the task. It was due to the main focus of the present research on the hemodynamic analysis and trend evaluation of these parameters to support the training effect.

Starting from this first evidence, firstly future studies should better monitor the modifications of the electrophysiological parameters (in terms of microvolts levels) during the task, discussing their trend for learning. Secondly, the connectivity patterns related to the NF training should be better explored in a way to disclose the underlying mechanisms related to brain plasticity. Also, different kinds of NF training could be compared in a way to find the most efficient way to induce emotional regulation. Finally, a clinical assessment about the generalization of the NF effects should be included, in a way to validate the positive impact found for at both behavioral and neural level.

\section{Author Disclosure}

Authors have no grants, financial interests, or conflicts to disclose.

\section{References}

American Psychiatric Association. (2000). Diagnostic and Statistical Manual of Mental Disorders (4th ed., text rev.). Washington, DC: Author.

Balconi, M., Brambilla, E., \& Falbo, L. (2009). BIS/BAS, cortical oscillations and coherence in response to emotional cues. Brain Research Bulletin, 80(3), 151-157. https://doi.org /10.1016/j.brainresbull.2009.07.001

Balconi, M., Cotelli, M., Rossi, R., Rillosi, L., Beneduce, R., Tura G. B., ... Vanutelli, M. E. (2018). Emotion regulation in Schizophrenia: A comparison between implicit (EEG and fNIRS) and explicit (valence) measures: Preliminary observations. Asian Journal of Psychiatry, 34, 12-13. https://doi.org/10.1016/j.ajp.2018.03.018

Balconi, M., Frezza, A., \& Vanutelli, M. E. (2018). Emotion regulation in schizophrenia: A pilot clinical intervention as 
assessed by EEG and optical imaging (functional near-infrared spectroscopy). Frontiers in Human Neuroscience, 12, 395. https://doi.org/10.3389/fnhum.2018.00395

Balconi, M., Grippa, E., \& Vanutelli, M. E. (2015a). Resting lateralized activity predicts the cortical response and appraisal of emotions: An fNIRS study. Social Cognitive and Affective Neuroscience, 10(12), 1607-1614. https://doi.org /10.1093/scan/nsv041

Balconi, M., Grippa, E., \& Vanutelli, M. E. (2015b). What hemodynamic (fNIRS), electrophysiological (EEG) and autonomic integrated measures can tell us about emotional processing. Brain and Cognition, 95, 67-76. https://doi.org /10.1016/j.bandc.2015.02.001

Balconi, M., Tirelli, S., \& Frezza, A. (2015). Event-related potentials (ERPs) and hemodynamic (functional near-infrared spectroscopy, fNIRS) as measures of schizophrenia deficits in emotional behavior. Frontiers in Psychology, 6, 1686. https://doi.org/10.3389/fpsyg.2015.01686

Balconi, M., \& Vanutelli, M. E. (2016). Emotions and BIS/BAS components affect brain activity (ERPs and fNIRS) in observing intra-species and inter-species interactions. Brain Imaging and Behavior, 10(3), 750-760. https://doi.org /10.1007/s11682-015-9443-z

Balconi, M, \& Vanutelli, M. E. (2017). Empathy in negative and positive interpersonal interactions. What is the relationship between central (EEG, fNIRS) and peripheral (autonomic) neurophysiological responses? Advances in Cognitive Psychology, 13(1), 105-120. https://doi.org/10.5709/acp0211-0

Balconi, M., Vanutelli, M. E., Bartolo, A., \& Cortesi, L. (2015). Transitive and intransitive gesture execution and observation compared to resting state: the hemodynamic measures (fNIRS). Cognitive Processing, 16(Suppl. 1), 125-129. https://doi.org/10.1007/s10339-015-0729-2

Balconi, M., Vanutelli, M. E., \& Grippa, E. (2017). Resting state and personality component (BIS/BAS) predict the brain activity (EEG and fNIRS measure) in response to emotional cues. Brain and Behavior, 7(5), e00686. https://doi.org /10.1002/brb3.686

Blanchard, J. J., \& Cohen, A. S. (2006). The structure of negative symptoms within schizophrenia: implications for assessment. Schizophrenia Bulletin, 32, 238-245. https://doi.org/10.1093 /schbul/sbj013

Bradley, M. M., \& Lang, P. J. (2007). Emotion and motivation. In J. T. Cacioppo, L. G. Tassinary, \& G. G. Berntson (Eds.), Handbook of psychophysiology (pp. 581-607). New York, NY: Cambridge University Press. https://doi.org/10.1017 /CBO9780511546396.025

Doi, H., Nishitani, S., \& Shinohara, K. (2013). NIRS as a tool for assaying emotional function in the prefrontal cortex. Frontiers of Human Neuroscience, 7, 770. https://doi.org/10.3389 /fnhum.2013.00770

Egashira, K., Matsuo, K., Nakashima, M., Watanuki, T., Harada, K., Nakano, M., ...Watanabe, Y. (2015). Blunted brain activation in patients with schizophrenia in response to emotional cognitive inhibition: A functional near-infrared spectroscopy study. Schizophrenia Research, 162(1-3), 196-204. https://doi.org/10.1016/j.schres.2014.12.038

Ehlis, A.-C., Herrmann, M. J., Plichta, M. M., \& Fallgatter, A. J. (2007). Cortical activation during two verbal fluency tasks in schizophrenic patients and healthy controls as assessed by multi-channel near-infrared spectroscopy. Psychiatry Research: Neuroimaging, 156(1), 1-13. https://doi.org /10.1016/j.pscychresns.2006.11.007

Fallgatter, A. J, \& Strik, W. K. (2000). Reduced frontal functional asymmetry in schizophrenia during a Cued Continuous Performance Test assessed with near-infrared spectroscopy. Schizophrenia Bulletin, 26(4), 913-919. https://doi.org /10.1093/oxfordjournals.schbul.a033505
Gruzelier, J., Hardman, E., Wild, J., \& Zaman, R. (1999). Learned control of slow potential interhemishpheric asymmetry in schizophrenia. International Journal of Psychophysiology, 34(3), 341-348. https://doi.org/10.1016/S01678760(99)00091-4

Herrmann, M. J., Huter, T., Plichta, M. M., Ehlis, A.-C., Alpers, G. W., Mühlberger, A., \& Fallgatter, A. J. (2008). Enhancement of activity of the primary visual cortex during processing of emotional stimuli as measured with event-related functional near-infrared spectroscopy and event-related potentials. Human Brain Mapping, 29(1) 28-35. https://doi.org/10.1002 /hbm.20368

Horan, W. P., Green, M. F., DeGroot, M., Fiske, A., Hellemann, G., Kee, K., \& Nuechterlein, K. H. (2012). Social cognition in schizophrenia, part 2: 12-month stability and prediction of functional outcome in first-episode patients. Schizophrenia Bulletin, 38(4), 865-872. https://doi.org/10.1093 /schbul/sbr001

Jobsis, F. F. (1977). Noninvasive, infrared monitoring of cerebral and myocardial oxygen sufficiency and circulatory parameters. Science, 198(4323), 1264-1267. https://doi.org /10.1126/science.929199

Kouijzer, M. E. J., van Schie, H. T., de Moor, J. M. H., Gerrits, B. J. L., \& Buitelaar, J. K. (2010). Neurofeedback treatment in autism. Preliminary findings in behavioral, cognitive, and neurophysiological functioning. Research in Autism Spectrum Disorders, 4(3), 386-399. $\quad$ https://doi.org/10.1016 /j.rasd.2009.10.007

Kring, A. M., \& Moran, E. K. (2008). Emotional response deficits in schizophrenia: Insights from affective science. Schizophrenia Bulletin, 34(5), 819-834. https://doi.org $/ 10.1093 /$ schbul/sbn071

Kubota, Y., Toichi, M., Shimizu, M., Mason, R. A., Coconcea, C. M., Findling, R. L., ... Calabrese, J. R. (2005). Prefrontal activation during verbal fluency tests in schizophrenia-A near-infrared spectroscopy (NIRS) study. Schizophrenia Research, 77(1), 65-73. https://doi.org/10.1016/j.schres.2005.01.007

Linden, D. E. J., \& Fallgatter, A. J. (2009). Neuroimaging in psychiatry: from bench to bedside. Frontiers in Human Neuroscience, $\quad 3, \quad 49 . \quad$ https://doi.org/10.3389 /neuro.09.049.2009

Naimijoo, P., Rezaei, O., \& Feizzadeh, G. (2015). Neurofeedback training in schizophrenia: A study on executive functioning. European Online Journal of Natural and Social Sciences, 4(1), 106-116.

Nan, W., Wan, F., Chang, L., Pun, S. H., Vai, M. I., \& Rosa, A. (2017). An exploratory study of intensive neurofeedback training for schizophrenia. Behavioural Neurology, 2017, 6914216. https://doi.org/10.1155/2017/6914216

NIRScout system [Apparatus]. Los Angeles, CA: NIRx Medical Technologies, LLC

nirsLAB (Version 2014.05) [Computer software]. (n.d.). Glen Head, NY: NIRx Medical Technologies, LLC.

Nishimura, Y., Takizawa, R., Muroi, M., Marumo, K., Kinou, M., \& Kasai, K. (2011). Prefrontal cortex activity during response inhibition associated with excitement symptoms in schizophrenia. Brain Research, 1370, 194-203. https://doi.org/10.1016/j.brainres.2010.11.003

ProComp2 2-channel biofeedback \& neurofeedback system with BioGraph Infiniti software [Apparatus]. Montreal, Canada: Thought Technology Ltd.

Pu, S., Nakagome, K., Yamada, T., Itakura, M., Satake, T., Ishida, H., ... Kaneko, K. (2013). Association between cognitive insight and prefrontal function during a cognitive task in schizophrenia: A multichannel near-infrared spectroscopy study. Schizophrenia Research, 150(1), 81-87. https://doi.org /10.1016/j.schres.2013.07.048

Pu, S., Nakagome, K., Yamada, T., Itakura, M., Yamanashi, T., Yamada, S., ... Kaneko, K. (2016). Social cognition and prefrontal hemodynamic responses during a working memory 
task in schizophrenia. Scientific Reports, 6, 22500. https://doi.org/10.1038/srep22500

Ruiz, S., Lee, S., Soekadar, S. R., Caria, A., Veit, R., Kircher, T., ... Sitaram, R. (2013). Acquired self-control of insula cortex modulates emotion recognition and brain network connectivity in schizophrenia. Human Brain Mapping, 34(1), 200-212. https://doi.org/10.1002/hbm.21427

Russell, J. A. (2003). Core affect and the psychological construction of emotion. Psychological Review, 110(1), 145172. https://doi.org/10.1037/0033-295X.110.1.145

Schneider, F., Rockstroh, B., Heimann, H., Lutzenberger, W., Mattes, R., Elbert, T., ... Bartels, M. (1992). Self-regulation of slow cortical potentials in psychiatric patients: Schizophrenia. Biofeedback and Self-regulation, 17(4), 277-292. https://doi.org/10.1007/BF01000051

Schummer, G. J. (2008). The Disconnection Syndrome. Biofeedback, 36(4), 157-162.

Sherlin, L. H., Arns, M., Lubar, J., Heinrich, H., Kerson, C., Strehl, U., \& Sterman, M. B. (2011) Neurofeedback and basic learning theory: Implications for research and practice. Journal of Neurotherapy, 15(4), 292-304. https://doi.org $/ 10.1080 / 10874208.2011 .623089$

Shinba, T., Nagano, M., Kariya, N., Ogawa, K., Shinozaki, T., Shimosato, S., \& Hoshi, Y. (2004). Near-infrared spectroscopy analysis of frontal lobe dysfunction in schizophrenia. Biological Psychiatry, 55(2), 154-164. https://doi.org /10.1016/S00063223(03)00547-X

Shoji, Y., Morita, K., Mori, K., Yamamoto, H., Fujiki, R., Ishii, Y., \& Uchimura, N. (2013). Characteristics of single event-related cerebral hemodynamics during verbal task in emotionally charged state measured by multi-channel near-infrared spectroscopy (NIRS) in patients with schizophrenia: Comparison with healthy subjects. Seishin Shinkeigaku Zasshi, 115(8), 853-862.

STIM2 [Computer software]. Charlotte, NC: Compumedics Neuroscan.

Surmeli, T., Ertem, A., Eralp, E., \& Kos, I. H. (2012). Schizophrenia and the efficacy of qEEG-guided neurofeedback treatment: a clinical case series. Clinical EEG and Neuroscience, 43(2), 133-44. https://doi.org/10.1177/1550059411429531

Takeshi, K., Nemoto, T., Fumoto, M., Arita, H., \& Mizuno, M. (2010) Reduced prefrontal cortex activation during divergent thinking in schizophrenia: A multi-channel NIRS study. Progress in Neuro-Psychopharmacology and Biological Psychiatry, 34(7), 1327-1332. https://doi.org/10.1016 /j.pnpbp.2010.07.021

Takizawa, R., Kasai, K., Kawakubo, Y., Marumo, K., Kawasaki, S., Yamasue, H., \& Fukuda, M. (2008). Reduced frontopolar activation during verbal fluency task in schizophrenia: A multichannel near-infrared spectroscopy study. Schizophrenia Reseach, 99(1-3), 250-262. https://doi.org/10.1016 /j.schres.2007.10.025

Taylor, S. F., Kang, J., Brege, I. S., Tso, I. F., Hosanagar, A., \& Johnson, T. D. (2012). Meta-analysis of functional neuroimaging studies of emotion perception and experience in schizophrenia. Biological Psychiatry, 71(2), 136-145. https://doi.org/10.1016/j.biopsych.2011.09.007

Watanabe, A., \& Kato, T. (1999). Cerebrovascular response to cognitive tasks in patients with schizophrenia measured by near-infrared spectroscopy. Schizophrenia Bulletin, 30(2), 435-444. /oxfordjournals.schbul.a007090

Watanabe, Y., Urakami, T., Hongo, S., \& Ohtsubo, T. (2015) Frontal lobe function and social adjustment in patients with schizophrenia: near-infrared spectroscopy. Human Psychopharmacoly: Clinical and Experimental. 30(1), 28-41. https://doi.org/10.1002/hup.2448

Williams, L. M., Das, P., Harris, A. W. F., Liddell, B. B., Brammer, M. J., Olivieri, G., ... Gordon, E. (2004). Dysregulation of arousal and amygdala-prefrontal systems in paranoid schizophrenia. The American Journal of Psychiatry, 161(3), 480-489. https://doi.org /10.1176/appi.ajp.161.3.480

Zhu, Y., Liu, X., Wang, H., Jiang, T., Fang, Y., Hu, H., ... Zhang, K. (2010). Reduced prefrontal activation during Tower of London in first-episode schizophrenia: A multi-channel near-infrared spectroscopy study. Neuroscience Letters, 478(3), 136-140. https://doi.org/10.1016/j.neulet.2010.05.003

Received: April 4, 2019

Accepted: April 28, 2019

Published: June 26, 2019 